\title{
The Impact of Severe Obesity on Post-Acute Rehabilitation Efficiency, Length of Stay, and Hospital Costs
}

\author{
Raj S. Padwal, ${ }^{1}$ Xiaoming Wang, ${ }^{1}$ Arya M. Sharma, ${ }^{1}$ and David Dyer ${ }^{2}$ \\ ${ }^{1}$ Department of Medicine, University of Alberta, Edmonton, AB, Canada T6G $2 B 7$ \\ ${ }^{2}$ Glenrose Rehabilitation Hospital, Alberta Health Services, Edmonton, AB, Canada T5G 0B7
}

Correspondence should be addressed to Raj S. Padwal, rpadwal@ualberta.ca

Received 3 November 2011; Accepted 17 December 2011

Academic Editor: Francesco Saverio Papadia

Copyright ( 2012 Raj S. Padwal et al. This is an open access article distributed under the Creative Commons Attribution License, which permits unrestricted use, distribution, and reproduction in any medium, provided the original work is properly cited.

\begin{abstract}
Background and Objective. The purpose of this retrospective observational study was to examine the influence of severe obesity on length of stay (LOS), rehabilitation efficiency, and hospital costs post-acute rehabilitation in a population-based, tertiary care, publicly-funded regional rehabilitation center. Participants. 42 severely obese subjects (mean age $53 \mathrm{y} ;$ mean BMI $50.9 \mathrm{~kg} / \mathrm{m}^{2}$ ) and 42 nonobese controls (mean age 59 y; mean BMI $23.0 \mathrm{~kg} / \mathrm{m}^{2}$ ) matched by sex and admitting diagnosis. Main Outcome Measures. Total LOS, rehab LOS, waiting for transfer LOS, Fuctional Independence Measure (FIM) efficiency, and hospital costs. Results. Compared to controls, severely obese subjects experienced longer total LOS (98.4 vs. 37.4 days; $P=0.03$ ), rehabilitation LOS (55.8 vs. 37.4 days; $P=0.04$ ), and waiting for transfer LOS ( 42.6 vs. 0 days; $P=0.006$ ); increased hospital costs $(\$ 115,822$ vs. $\$ 43,969 ; P=0.03)$; and similar FIM efficiency $(0.58$ vs. $0.67 ; P=0.27)$. Severe obesity was an independent predictor of total LOS (beta-coefficient $0.51 ; P=0.03)$, rehab LOS $(0.46 ; P=0.02)$ but not FIM efficiency $(-0.63 ; P=0.06)$. Conclusion. Severe obesity adversely affects rehabilitation LOS and expenditures. Targeted interventions in severely obese individuals to optimize post-acute rehabilitation care delivery are needed.
\end{abstract}

\section{Introduction}

Following acute medical illness, traumatic injury, or surgery, patients often require rehabilitative care designed to improve physical, cognitive, and functional status and reduce caregiver burden [1]. Demand for this type of care, often termed "post-acute rehabilitation" or PAR, is rising, in part because the types of patients commonly referred for PAR (elderly, frail, medically complex) are becoming increasingly prevalent [1]. Thus, maximizing the efficiency and costeffectiveness of PAR in this era of heightened competition for increasingly scarce health care resources is a major priority.

Severe or Class III obesity (body mass index or BMI $\geq$ $40 \mathrm{~kg} / \mathrm{m}^{2}$ ) affects $3 \%$ of the adult Canadian population and has tripled in prevalence over three decades [2, 3]. The long-term health consequences of Class III obesity are well documented and include premature mortality; chronic medical illness; increased health care costs; dramatically reduced health-related quality of life (QOL) $[4,5]$. In contrast, the impact of severe obesity on PAR outcomes has not been well studied even though obese individuals are more likely than their normal weight counterparts to be frail, functionally disabled, and medically complex [5-7]. A greater understanding of this relationship will help plan service delivery needs if anticipated increases in the number of severely obese patients accessing PAR occur.

Two previous studies examining PAR after hip and knee arthroplasty reported higher costs and less efficient rehabilitation in severely obese subjects compared to either overweight or nonobese controls $[8,9]$. However, these data have limited generalizability because they were both performed in private health care settings and focused solely on orthopedic PAR. Another US study examining deconditioned general medical patients reported no difference in rehabilitation efficiency in severely compared to normal weight subjects [10].

The objective of this study was to examine the impact of severe obesity on length of stay (LOS), functional status change, and inpatient costs in a population-based, publicly funded, tertiary care regional Canadian rehabilitation center. We hypothesized that severe obesity would be associated with reduced rehabilitation efficiency, greater lengths of stay, and higher costs during PAR compared to controls. 


\section{Methods}

2.1. Setting. This retrospective analysis examined data from the Glenrose Rehabilitation Hospital (GRH), the largest freestanding academic tertiary-care rehabilitation center in Canada. The GRH delivers comprehensive rehabilitation services to patients from Alberta, Western Arctic, British Columbia, and Northwestern Saskatchewan serving a catchment area of over 9 million people and treating over 1,800 patients per year.

2.2. Rehabilitation Program. The Glenrose Rehabilitation Hospital provides specialized comprehensive rehabilitation for children, adults, and older adults with physical and or cognitive disability. Coordinated services including assessment, therapeutic interventions, medical interventions, counseling, and patient and family education are all provided. Direct and indirect client services are provided by all or a combination of 16 clinical disciplines. Direct care is available six days per week and includes physical rehabilitation therapy delivered by physical therapists, occupational therapists, speech language pathologists, and dietitians as well as specialty trained rehabilitation nurses. Indirect care includes services such as aiding and advising rehabilitation clients and families on issues including discharge planning, home modifications, and financial planning. The goals of the programs are to optimize the client's ability to regain and maintain his or her functional abilities to resume their desired lifestyle back in the community.

2.3. Data Collection. A prospectively collected electronic registry for all GRH inpatients containing admission body weight grouped into three categories (<90 kg, 90-136 kg, and $\geq 137 \mathrm{~kg}$ ) was queried identify adult patients (age $\geq 18$ years) admitted between April 1, 2004 and March 31, 2009 with a body weight over $137 \mathrm{~kg}$. Next, $1: 1$ controls with body weights $<90 \mathrm{~kg}$ matched on sex, admission diagnosis (orthopaedic, medical, or brain/spinal cord injury), and admission date (within 2 years) were randomly selected. Chart reviews were then performed to ascertain height and calculate BMI. Cases with BMI levels $<40 \mathrm{~kg} / \mathrm{m}^{2}(n=2)$ or who died in hospital $(n=2)$ were excluded.

Additional sociodemographic and clinical data were collected for each subject, including age, marital status (married or common law, single, or widowed), employment status (employed, unemployed or social assistance, retired), and the following medical comorbidities (coded as binary variables): hypertension, diabetes, dyslipidemia, stroke, heart disease, peripheral vascular disease, chronic obstructive pulmonary disease, asthma, sleep apnea, cancer, arthritis, dementia, smoking, mental health, neurologic disease, renal failure, venous thromboembolic disease, and presence of a pressure ulcer.

2.4. Functional Status Assessment. Functional status was assessed using the Functional Independence Measure (FIM) tool [11]. The FIM is a valid, reliable, and sensitive measure of disability severity and burden of care in the inpatient clinical rehabilitation setting and is widely used to monitor rehabilitation progress over the course of an admission $[12,13]$. Eighteen activities of daily living reflecting both motor and cognitive disability are rated on a 7-point scale ranging from fully independent (7) to fully dependent (1). Summation of the scores across the 18 items reflects overall burden of care [14]. Possible overall scores range from 18 to 126 , with higher scores indicating greater functional independence. The FIM can be subdivided into a 13-item motor domain and a 5-item cognitive domain.

Trained and certified data collectors recorded and calculated FIM scores at admission and discharge in all patients. FIM efficiency was calculated by dividing the change in FIM score from admission to discharge by the rehabilitation LOS (see below). Motor and cognitive subscores were also calculated.

2.5. Length-of-Stay Measurement. Patient-specific identifiers were used to perform data linkage with Canadian Institute for Health Information (CIHI) administrative data to ascertain LOS. CIHI collects data from inpatient facilities across Canada (excluding Quebec) [15]. Extensive quality control measures ensure high-quality data collection within CIHI inpatient data; the frequency of missing data elements is less than $0.001 \%$ [15].

Overall LOS was subdivided into the rehabilitation LOS and waiting-transfer-of-service (WTS) LOS. Rehabilitation LOS measured the duration of active rehabilitation and WTS LOS, measured the time period between the completion of active rehabilitation and discharge from the hospital. Patients accumulate WTS LOS days if they are awaiting home environment modification prior to discharge or have been unable to progress to independence and require placement in a long-term care facility.

2.6. Costs. Total hospital costs were calculated for severely obese subjects and controls by multiplying the average overall length of stay for each study group by $\$ 1177 \mathrm{Cdn}$, the average daily cost of an inpatient stay at GRH.

2.7. Statistical Analysis. The means and proportions of baseline variables, including FIM scores, FIM efficiency, LOS, and total costs were compared between cases and controls using Wilcoxon rank sum tests. Results are reported for the overall population and according to each of the three admitting diagnostic groups.

Multivariable linear regression was used to determine the independent predictors of overall LOS, rehab LOS, WTS LOS, and FIM efficiency in the overall population. Log transformation of each outcome was necessary to satisfy model assumptions. Age and study group were first forced into all models. As the patients were matched based on sex and admission diagnosis, these were not entered into the models. Additional covariates with a $P$ value $<0.25$ on univariable analysis were also considered in the initial model. Potential model covariates included age, sex, marital status (married versus single/widowed), employment status 
TABLE 1: Baseline characteristics.

\begin{tabular}{|c|c|c|c|}
\hline Variable & Severe obesity $(n=42)$ & $\begin{array}{l}\text { Controls } \\
(N=42) \\
\end{array}$ & $P$ value \\
\hline Sex, male, no $(\%)^{*}$ & $32(76)$ & $32(76)$ & - \\
\hline Age, yr, mean (SD) & $53(15)$ & $59(14)$ & 0.06 \\
\hline Weight, kg, mean (SD) & $158.8(25.3)$ & $69.3(12.4)$ & $<0.0001$ \\
\hline Height, cm, mean (SD) & $177.6(10.2)$ & $173.1(10.3)$ & 0.05 \\
\hline Body mass index, $\mathrm{kg} / \mathrm{m}^{2}$, mean $(\mathrm{SD})$ & $50.9(8.3)$ & $23.0(3.0)$ & $<0.0001$ \\
\hline \multicolumn{4}{|l|}{ Reason for requiring rehabilitation, no $(\%)^{*}$} \\
\hline Orthopaedic surgery & $26(62)$ & $26(62)$ & - \\
\hline Acute medical illness & $8(19)$ & $8(19)$ & - \\
\hline Stroke, brain or spinal cord injury & $8(19)$ & $8(19)$ & - \\
\hline Married or common law & $18(43)$ & $19(45)$ & 0.83 \\
\hline Single or widow & $24(57)$ & $23(55)$ & 0.83 \\
\hline \multicolumn{4}{|l|}{ Employment status, no (\%) } \\
\hline Employed & $13(31)$ & $17(40)$ & 0.36 \\
\hline Unemployed or social assistance & $13(31)$ & $11(26)$ & 0.63 \\
\hline Retired & $16(38)$ & $14(33)$ & 0.65 \\
\hline \multicolumn{4}{|l|}{ Smoking, no (\%) } \\
\hline Never & $18(43)$ & $15(36)$ & 0.50 \\
\hline Current & $5(12)$ & $13(31)$ & 0.03 \\
\hline Former & $19(45)$ & $14(33)$ & 0.26 \\
\hline Hypertension & $39(92)$ & $19(45)$ & $<0.0001$ \\
\hline Diabetes, no (\%) & $36(86)$ & $12(29)$ & $<0.0001$ \\
\hline Dyslipidemia, no (\%) & $19(45)$ & $12(29)$ & 0.11 \\
\hline Stroke, no $(\%)$ & $9(21)$ & $7(17)$ & 0.58 \\
\hline Heart disease, no (\%) & $16(38)$ & $8(19)$ & 0.05 \\
\hline Peripheral vascular disease, no (\%) & $6(14)$ & $5(12)$ & 0.75 \\
\hline Chronic obstructive pulmonary disease, no (\%) & $5(12)$ & $11(26)$ & 0.10 \\
\hline Asthma, no (\%) & $3(7)$ & $0(0)$ & 0.08 \\
\hline Sleep apnea, no (\%) & $18(43)$ & $0(0)$ & $<0.0001$ \\
\hline Cancer, no (\%) & $0(0)$ & $3(7)$ & 0.08 \\
\hline Arthritis, no (\%) & $16(38)$ & $8(19)$ & 0.05 \\
\hline Dementia, no (\%) & $4(10)$ & $6(14)$ & 0.50 \\
\hline Mental illness, no (\%) & $20(48)$ & $12(29)$ & 0.07 \\
\hline Neurological disease, no (\%) & $10(24)$ & $3(7)$ & 0.03 \\
\hline Renal failure, no (\%) & $12(29)$ & $4(10)$ & 0.03 \\
\hline Pressure ulcer, no (\%) & $9(21)$ & $7(17)$ & 0.58 \\
\hline Venous thromboembolic disease, no (\%) & $4(10)$ & $3(7)$ & 0.69 \\
\hline
\end{tabular}

* Subjects were matched on sex and admitting diagnosis.

(employed, unemployed or social assistance, retired), reason for requiring rehabilitation (orthopedic surgery, acute medical illness, stroke or brain/spinal cord injury), smoking (never, former, current), and medical comorbidities (Table 1). The final model was created using a stepwise backwards selection method to determine which of these additional covariates contributed to the model at a Wald Chi-square $P$ value of 0.25. SAS (Version 9.2, Cary, NC) was used for all analyses. $P$ values less than 0.05 were considered statistically significant.
2.8. Ethics Approval. Ethics approval was obtained from the University of Alberta Research Ethics Board.

\section{Results}

3.1. Baseline Characteristics. The baseline sociodemographic characteristics of all 84 patients are detailed in Table 1. Sixtytwo percent of subjects were admitted after orthopaedic surgery, 19\% after admission for acute medical illness, and $19 \%$ following stroke, brain, or spinal cord injury. Subjects 
TABLE 2: Length of stay, functional independence measure, and cost outcomes.

\begin{tabular}{lcc}
\hline Outcome & Severe obesity $(n=42)$ & Controls $(N=42)$ \\
\hline Length of Stay & & $P$ value \\
Total LOS, days, mean (SD) & $98.4(186.3)$ & $37.4(20.9)$ \\
Rehab LOS, days, mean (SD) & $55.8(42.66)$ & $37.4(20.9)$ \\
WTS LOS, days, mean (SD) & $42.6(167.00)$ & $0(0)$ \\
Functional independent measure & & 0.03 \\
Total FIM score admission, mean (SD) & $85.5(16.6)$ & $106.8(19.9)$ \\
Total FIM score discharge, mean (SD) & $107.2(12.5)$ & $0.67(0.54)$ \\
Overall FIM efficiency, mean (SD) & $0.58(0.53)$ & $55.7(18.3)$ \\
FIM motor score on admission, mean (SD) & $54.1(14.3)$ & $76.2(13.9)$ \\
FIM motor score on discharge, mean (SD) & $74.4(11.6)$ & $20.0(13.3)$ \\
Change in motor score, mean (SD) & $20.2(14.7)$ & $29.7(8.4)$ \\
FIM cognitive score on admission, mean (SD) & $31.3(5.9)$ & $30.6(7.4)$ \\
FIM cognitive score on discharge, mean (SD) & $32.9(3.8)$ & $1.0(2.2)$ \\
Change in cognitive score, mean (SD) & $1.5(3.7)$ & 0.48 \\
Costs & & 0.54 \\
Total costs, mean (SD) & $115,822(219,314)$ & 0.41 \\
\hline
\end{tabular}

LOS: length of stay; FIM: functional independence measure; SD: standard deviation; WTS: waiting transfer of service.

were $76 \%$ male. Severely obese subjects were younger (53 versus 59 years old), heavier (BMI 50.9 versus $23.0 \mathrm{~kg} / \mathrm{m}^{2}$ ) and had a significantly higher prevalence of hypertension (93 versus $45 \%$ ), diabetes (86 versus $29 \%$ ), sleep apnea (43 versus 0\%), neurological disease (24 versus $7 \%$ ), and renal failure (29 versus $10 \%$ ) but a lower prevalence of current smoking (12 versus $31 \%$ ). The range of BMI values was $41-76 \mathrm{~kg} / \mathrm{m}^{2}$ in the severely obese patients and $17-27 \mathrm{~kg} / \mathrm{m}^{2}$ for controls.

3.2. LOS, FIM, and Cost Outcomes. Severely obese subjects had a significantly greater mean overall LOS (98.4 versus 37.4 days; $P=0.03$ ), rehab LOS (55.8 versus 37.4 days $P=0.04$ ), and WTS LOS (42.6 versus 0 days; $P=0.006$ ) compared to controls (Table 2). Admission and discharge FIM scores and FIM score changes were similar between study groups (Table 2). FIM efficiency was lower in severely obese subjects; this difference was not statistically significant $(0.58$ versus $0.67 ; P=0.27)$. FIM motor and cognitive subscore changes were nearly identical between severely obese subjects and controls (Table 2).

Total daily hospital costs were $\$ 115,822$ in the severely obese compared to $\$ 43,969$ in controls $(P=0.03)$.

LOS and FIM outcomes stratified by admitting diagnosis are presented in Table 3. LOS outcomes were consistently higher in the severely obese, but results were statistically significant only in the orthopedic group. FIM efficiency was not significantly different between the severely obese and controls in any of the subgroups.

3.3. Linear Regression Analysis. The results of separate covariate-adjusted linear regression models examining log overall LOS, log rehab LOS, and log FIM efficiency are detailed in Table 4. Severe obesity was an independent predictor of greater overall LOS (beta-coefficient $0.51 ; P=0.03$ ) and rehab LOS $(0.46 ; P=0.02)$. Severe obesity was associated with reduced FIM efficiency, but this association was not statistically significant $(-0.63 ; P=0.06)$.

\section{Discussion}

In summary, severely obese subjects undergoing PAR in a population-based, publically funded rehabilitation center experienced increased lengths of stay and incurred greater hospital costs but had similar rehabilitation efficiency compared to nonobese controls. To our knowledge, this is the first study to examine PAR outcomes in severely obese patients cared for within a publically funded health care system. Our findings should prompt clinicians and decision makers within our rehabilitation program and similarly structured programs to better understand the reasons for increased LOS and costs and develop strategies to mitigate these effects.

Few other studies have examined PAR outcomes in the severely obese and all have taken place within the US health care system. Two controlled observational studies of postorthopedic-surgery patients reported higher costs and lower FIM efficiency in severely obese subjects compared to nonobese controls $[8,9]$. LOS results in these two studies were inconsistent. In 177 patients undergoing PAR following hip arthroplasty at a university-affiliated teaching center, severely obese subjects had longer hospital stays compared to overweight patients ( 12.3 versus 11.8 days; $P<0.05$ ) [9]. Conversely, in 5428 patients across 15 sites undergoing PAR after total knee replacement, LOS was shorter in the severely obese compared to nonobese subjects (9.7 versus 10.3 days; $P<0.05$ ) [8]. A study of 1077 deconditioned general medical patients reported that FIM score gains were not significantly different between severely obese and normal weight subjects 
TABLE 3: Length of stay and functional independence measure outcomes by admitting diagnosis subgroup.

\begin{tabular}{|c|c|c|c|c|c|c|c|c|c|}
\hline \multirow[b]{2}{*}{ Outcome } & \multicolumn{3}{|c|}{ After orthopaedic surgery } & \multicolumn{3}{|c|}{ Medical illness } & \multicolumn{3}{|c|}{ Stroke/brain/spinal injury } \\
\hline & $\begin{array}{l}\text { Severe } \\
\text { obesity } \\
(n=26)\end{array}$ & $\begin{array}{l}\text { Controls } \\
(n=26)\end{array}$ & $P$ value & $\begin{array}{l}\text { Severe } \\
\text { obesity } \\
(N=8)\end{array}$ & $\begin{array}{l}\text { Controls } \\
(n=8)\end{array}$ & $P$ value & $\begin{array}{l}\text { Severe } \\
\text { obesity } \\
(N=8)\end{array}$ & $\begin{array}{l}\text { Controls } \\
(n=8)\end{array}$ & $P$ value \\
\hline $\begin{array}{l}\text { Total LOS, day, } \\
\text { mean (SD) }\end{array}$ & $\begin{array}{c}120.5 \\
(225.3)\end{array}$ & $37.3(22.4)$ & 0.02 & $46.5(25.9)$ & $35.0(19.2)$ & 0.43 & $\begin{array}{c}78.6 \\
(126.7)\end{array}$ & $39.8(19.9)$ & 0.83 \\
\hline $\begin{array}{l}\text { Rehab LOS, day, } \\
\text { mean (SD) }\end{array}$ & $62.3(47.3)$ & $37.3(22.4)$ & 0.02 & $42.5(15.7)$ & $35.0(19.2)$ & 0.43 & $48.1(44.8)$ & $39.8(19.9)$ & 0.83 \\
\hline $\begin{array}{l}\text { WTS LOS, day, } \\
\text { mean (SD) }\end{array}$ & $\begin{array}{c}58.2 \\
(207.0)\end{array}$ & $0.0(0.0)$ & 0.02 & $4.0(11.3)$ & $0.0(0.0)$ & 0.32 & $30.5(86.3)$ & $0.0(0.0)$ & 0.32 \\
\hline $\begin{array}{l}\text { FIM score } \\
\text { admission, mean } \\
\text { (SD) }\end{array}$ & $88.9(15.3)$ & $94.4(15.2)$ & 0.21 & $84.8(16.4)$ & $88.3(13.0)$ & 0.56 & $75.9(18.5)$ & $54.9(28.8)$ & 0.14 \\
\hline $\begin{array}{l}\text { FIM score } \\
\text { discharge, mean } \\
(\mathrm{SD})\end{array}$ & $\begin{array}{l}109.0 \\
(10.7)\end{array}$ & $114.0(4.1)$ & 0.12 & $107.3(7.8)$ & $112.1(5.2)$ & 0.34 & $\begin{array}{l}103.0 \\
(19.7)\end{array}$ & $78.4(32.9)$ & 0.03 \\
\hline $\begin{array}{l}\text { FIM efficiency, } \\
\text { mean (SD) }\end{array}$ & $0.49(0.49)$ & $0.66(0.62)$ & 0.28 & $0.56(0.40)$ & $0.78(0.47)$ & 0.21 & $0.88(0.68)$ & $0.62(0.32)$ & 0.34 \\
\hline
\end{tabular}

LOS: length of stay; FIM: functional independence measure; SD: standard deviation; WTS: waiting transfer of service.

TABLE 4: Linear regression analysis examining the effect of severe obesity on log LOS and log FIM efficiency.

\begin{tabular}{|c|c|c|c|}
\hline Model & Variable & Beta-coefficient (SE) & $P$ value \\
\hline \multirow{6}{*}{$\begin{array}{l}\text { Overall LOS } \\
\text { model } R^{2}=0.1798\end{array}$} & Intercept & $4.44(0.46)$ & $<0.0001$ \\
\hline & Severe obesity & $0.51(0.23)$ & 0.03 \\
\hline & Retired (versus employed) & $0.67(0.26)$ & 0.01 \\
\hline & Age (per one year increase) & $-0.02(0.009)$ & 0.04 \\
\hline & smoking former & $-0.26(0.19)$ & 0.17 \\
\hline & Diabetes & $-0.26(0.22)$ & 0.25 \\
\hline \multirow{6}{*}{$\begin{array}{l}\text { Rehab LOS } \\
\text { model } R^{2}=0.1693\end{array}$} & Intercept & $4.14(0.37)$ & $<0.0001$ \\
\hline & Severe obesity & $0.46(0.18)$ & 0.02 \\
\hline & Retired (versus employed) & $0.47(0.21)$ & 0.03 \\
\hline & Diabetes & $-0.32(0.18)$ & 0.08 \\
\hline & Age (per one year increase) & $-0.01(0.01)$ & 0.09 \\
\hline & Mental illness & $-0.21(0.15)$ & 0.16 \\
\hline \multirow{13}{*}{$\begin{array}{l}\text { FIM efficiency } \\
\text { model } R^{2}=0.3133\end{array}$} & Intercept & $-2.12(0.59)$ & 0.0006 \\
\hline & Severe obesity & $-0.63(0.33)$ & 0.06 \\
\hline & Unemployed (versus employed) & $-0.84(0.27)$ & 0.003 \\
\hline & Heart Disease & $-0.60(0.34)$ & 0.08 \\
\hline & Mental illness & $0.65(0.25)$ & 0.01 \\
\hline & Asthma & $1.01(0.63)$ & 0.11 \\
\hline & Current smoker (versus never) & $0.60(0.30)$ & 0.05 \\
\hline & Age & $0.02(0.01)$ & 0.07 \\
\hline & HTN & $0.52(0.32)$ & 0.10 \\
\hline & Venous thromboembolic disease & $0.68(0.42)$ & 0.13 \\
\hline & Dementia & $-0.66(0.42)$ & 0.13 \\
\hline & PVD & $-0.55(0.40)$ & 0.18 \\
\hline & $\mathrm{DM}$ & $0.37(0.30)$ & 0.23 \\
\hline
\end{tabular}

LOS: length of stay; FIM: functional independence measure. 
[10]. Other PAR studies have not specifically focused on severe obesity per se but, rather, have included patients with lesser degrees of adiposity. These studies have reported smaller FIM score gains in patients with excess adiposity undergoing PAR after spinal cord or burn injuries $[16,17]$.

Thus, when our results and the results of prior studies are considered in aggregate, PAR costs are consistently increased in the severely obese, but PAR efficiency and LOS findings are contradictory. Differences between studies in rehabilitation programs, underlying reasons for requiring PAR, prevalence of comorbid conditions and health care delivery models may account for some of these contradictory results. We also note that the BMI range selected for the control group differs between studies: normal (BMI $18.5-24.9 \mathrm{~kg} / \mathrm{m}^{2}$ ), nonoverweight $\left(\mathrm{BMI}<25 \mathrm{~kg} / \mathrm{m}^{2}\right.$ ), and overweight (BMI $25.0-29.9 \mathrm{~kg} / \mathrm{m}^{2}$ ) controls have all been used and this may partly account for the discrepant findings. The BMI level(s) associated with optimal values for PAR efficiency and LOS also requires further clarification. When examined across the range of BMI levels, the relationships between BMI and LOS, BMI and FIM efficiency, and BMI and costs were curvilinear after orthopedic surgery [9]. That is, LOS and costs were higher and FIM efficiency was lower in patients with either very low or very high BMI and optimal values for each of these outcomes were found at BMI levels of around $30 \mathrm{~kg} / \mathrm{m}^{2}$. In deconditioned medical patients, FIM score gains were greatest in the group with BMI levels between 30 and $35 \mathrm{~kg} / \mathrm{m}^{2}$. We hypothesize that the presence of sicker, frailer and nutritionally compromised patients within the lower BMI categories may result in worse PAR outcomes, although further study is required to clarify this issue.

Our LOS and FIM score change results were consistent when stratified by admitting diagnosis, but only the orthopedic surgery subgroup LOS results achieved statistical significance and this may have resulted from a lack of power due to the low sample sizes in the other two subgroups. We also found an increase in WTS LOS in the severely obese; in contrast, increased WTS LOS was not found in the control group. No prior study has reported this outcome. We suspect that the increased WTS LOS in the severely obese is a consequence of the patient's inability to gain independence following rehabilitation. In our experience, these subjects cannot return home and due to a lack of suitable alternative discharge destinations, often wait in hospital for transfer to a nursing home. Further study is required to determine the extent to which this issue contributes to a "bed blocking" effect (preventing use of these beds for other patients requiring PAR) and to reductions in cost-efficient health care delivery.

Targeted interventions to optimize PAR delivery and improve PAR efficacy in the severely obese may potentially lead to result in earlier discharge, reduced LOS, and decreased costs. Specifically, tailored interventions designed to improve the motor impairment, mobility disability, and frailty commonly found in the severely obese may improve outcomes $[6,7]$. One might consider modifying rehabilitation equipment so that traditional components of PAR can be safely delivered in the severely obese. Employing activities such as water-based exercises that "cushion" the excess bulk found in these subjects may make physical exercise easier and less stressful on joints. Multidisciplinary case management, with specific attention paid to the challenges and complexities found in severely obese subjects may also help to optimize care and preempt complications and setbacks.

However there are very limited published data on bariatric-specific PAR interventions and this deficiency was recently recognized at a multidisciplinary consensus conference [18]. Many potential barriers to developing effective rehabilitation strategies in bariatric patients were identified by this expert panel, including the lack of bariatricspecific rehabilitation programs in both acute and post-acute rehabilitation, a paucity of standards of care specific to obese patients, weight bias on the part of health care staff, and a lack of training programs and research consortia specializing in bariatric rehabilitation [18]. The higher prevalence of medical complexity, mental health impairment, and psychosocial dysfunction in the severely obese were also recognized as potential barriers to effective rehabilitation and factors that may increase the likelihood of acute illness relapse during PAR $[5,18]$.

A major strength of this study is the low risk of selection bias, as the GRH serves as the primary rehabilitation center for a large catchment area and, in contrast to privately funded rehabilitation centers, admission to the GRH is not influenced by payment or reimbursement factors. In addition, we did not limit our analysis to a specific type of PAR program; rather, we examined all severely patients admitted for PAR over a five-year span. Unlike other studies, we also were able to breakdown overall LOS into separate components to examine the effect of severe obesity on rehabilitation and nonrehabilitation LOS. However, a limitation of this study is that the sample size of this study was not large and this may have limited the statistical power of the study, particularly when performing subgroup analyses. A second limitation was that we only abstracted data on severely obese patients and nonobese controls and are were unable to compare the severely obese patients to patients with lesser degrees of obesity.

The need for PAR in severely obese patients will undoubtedly increase given the rising prevalence of severe obesity and expected increases in the need for PAR [1]. Data from this study will help clinicians and administrators estimate the effect of severe obesity on PAR in their institution allocate resources accordingly. A proactive approach to PAR in the severely obese is recommended and further research should focus on developing interventions to maximize efficient PAR delivery in this patient population.

\section{Conflict of Interests}

The authors declare no conflict of interests with respect to this work.

\section{Acknowledgments}

This study was unfunded. R. S. Padwal had full access to the data and takes responsibility for the integrity of the data and accuracy of the data analysis. R. S. Padwal and A. M. 
Sharma are supported by an alternative funding plan from the Government of Alberta and the University of Alberta. A. M. Sharma is supported by an Alberta Health Services Chair in Obesity Research and Management.

\section{References}

[1] A. W. Heinemann, "State of the science on postacute rehabilitation: setting a research agenda and developing an evidence base for practice and public policy: an introduction," Assistive Technology, vol. 20, no. 1, pp. 55-60, 2008.

[2] M. Shields, M. Carroll, and C. Ogden, "Adult obesity prevalence in Canada and the United States," NCHS Data Briefs, vol. 56 , pp. 1-8, 2011.

[3] M. Tjepkema, "Measured obesity. Adult obesity in Canada: measured height and weight," in Nutrition: Findings from the Canadian Community Health Survey 2004, Issue I (cat no 82620-MWE2005001), 2008, http://www.statcan.ca/english/research/82-620-MIE/2005001/articles/adults/aobesity.htm.

[4] A. Peeters, J. J. Barendregt, F. Willekens et al., "Obesity in adulthood and its consequences for life expectancy: a life-table analysis," Annals of Internal Medicine, vol. 138, no. 1, pp. 24 32, 2003.

[5] D. D. Hensrud and S. Klein, "Extreme obesity: a new medical crisis in the United States," Mayo Clinic Proceedings, vol. 81, supplement 10, pp. S5-S10, 2006.

[6] R. E. Hubbard, I. A. Lang, D. J. Llewellyn, and K. Rockwood, "Frailty, body mass index, and abdominal obesity in older people," Journals of Gerontology A, vol. 65, no. 4, pp. 377-381, 2010.

[7] D. T. Villareal, M. Banks, C. Siener, D. R. Sinacore, and S. Klein, "Physical frailty and body composition in obese elderly men and women," Obesity Research, vol. 12, no. 6, pp. 913920, 2004.

[8] H. K. Vincent and K. R. Vincent, "Obesity and inpatient rehabilitation outcomes following knee arthroplasty: a multicenter study," Obesity, vol. 16, no. 1, pp. 130-136, 2008.

[9] H. K. Vincent, J. P. Weng, and K. R. Vincent, "Effect of obesity on inpatient rehabilitation outcomes after total hip arthroplasty," Obesity, vol. 15, no. 2, pp. 522-530, 2007.

[10] N. B. Jain, S. Al-Adawi, A. S. S. Dorvlo, and D. T. Burke, "Association between body mass index and functional independence measure in patients with deconditioning," American Journal of Physical Medicine \& Rehabilitation, vol. 87, no. 1, pp. 21-25, 2008.

[11] Uniform Data System for Medical Rehabilitation website, 2011, http://www.udsmr.org.

[12] T. A. Dodds, D. P. Martin, W. C. Stolov, and R. A. Deyo, "A validation of the functional independence measurement and its performance among rehabilitation inpatients," Archives of Physical Medicine and Rehabilitation, vol. 74, no. 5, pp. 531536, 1993.

[13] M. E. Cohen and R. J. Marino, "The tools of disability outcomes research functional status measures," Archives of Physical Medicine and Rehabilitation, vol. 81, no. 12, part B, pp. S21-S29, 2000.

[14] M. G. Stineman, J. A. Shea, A. Jette et al., "The functional independence measure: tests of scaling assumptions, structure, and reliability across 20 diverse impairment categories," Archives of Physical Medicine and Rehabilitation, vol. 77, no. 11, pp. 1101-1108, 1996.
[15] Canadian Institute for Health Information, "Discharge Abstract Database," 2011, http://www.cihi.ca//CIHI-ext-portal/ pdf/internet/DAD_EXECUTIVE_SUM_09_10_EN.

[16] K. W. Stenson, A. Deutsch, A. W. Heinemann, and D. Chen, "Obesity and inpatient rehabilitation outcomes for patients with a traumatic spinal cord injury," Archives of Physical Medicine and Rehabilitation, vol. 92, no. 3, pp. 384-390, 2011.

[17] R. T. Farrell, R. L. Gamelli, R. F. Aleem, and J. M. Sinacore, "The relationship of body mass index and functional outcomes in patients with acute burns," Journal of Burn Care and Research, vol. 29, no. 1, pp. 102-108, 2008.

[18] M. Forhan, Y. Bhambhani, D. Dyer, X. Ramos-Salas, M. Ferguson-Pell, and A. Sharma, "Rehabilitation in bariatrics: opportunities for practice and research," Disability and Rehabilitation, vol. 32, no. 11, pp. 952-959, 2010. 


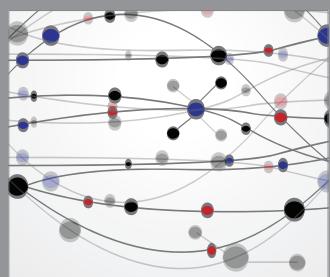

The Scientific World Journal
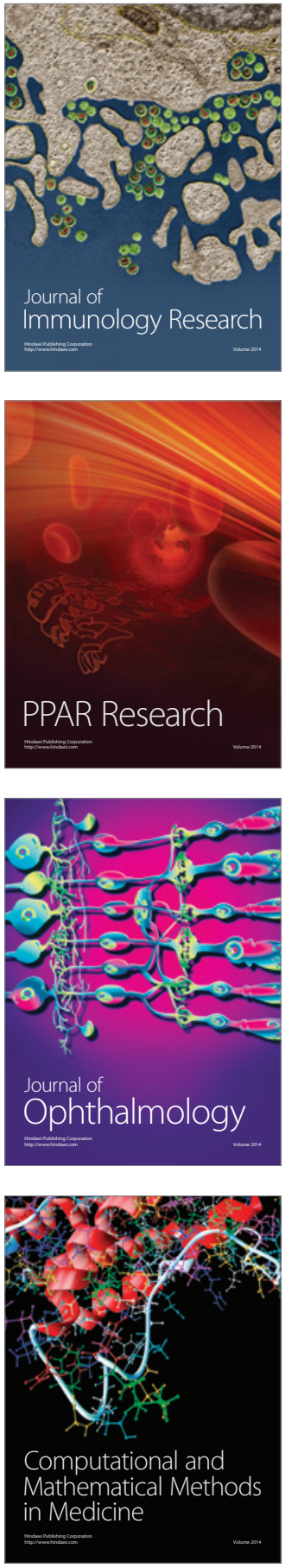

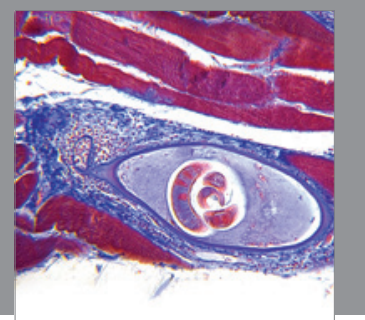

Gastroenterology

Research and Practice
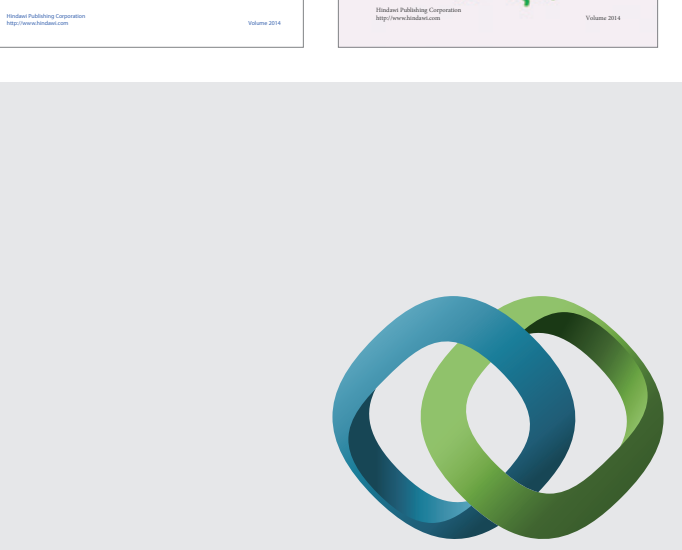

\section{Hindawi}

Submit your manuscripts at

http://www.hindawi.com
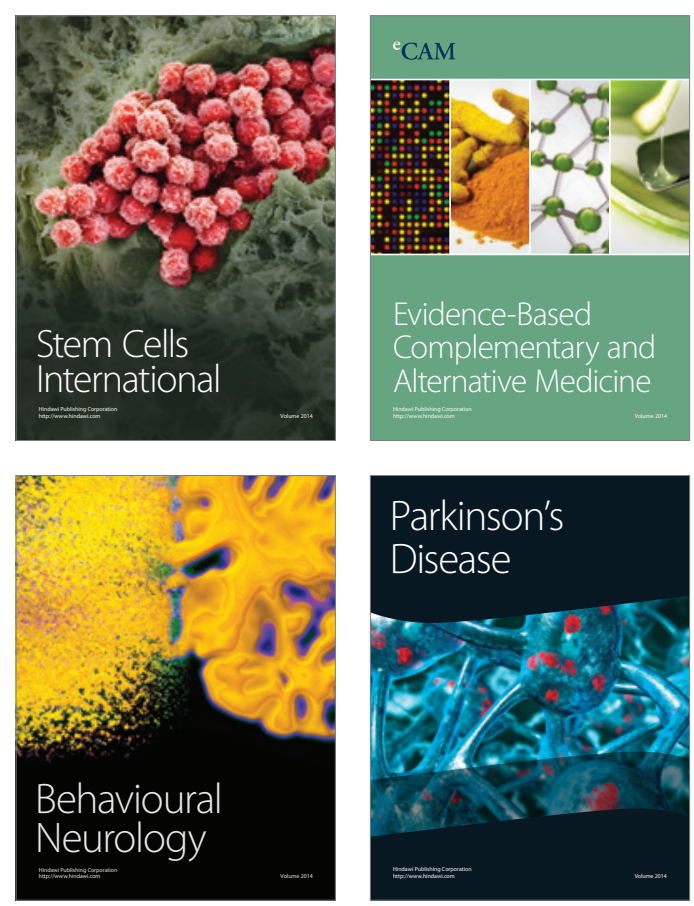

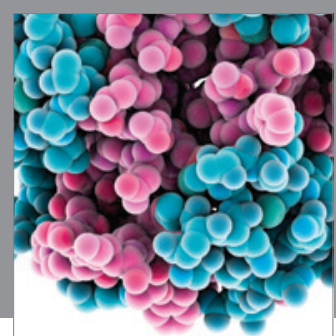

Journal of
Diabetes Research

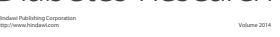

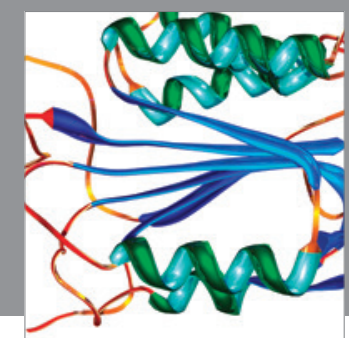

Disease Markers
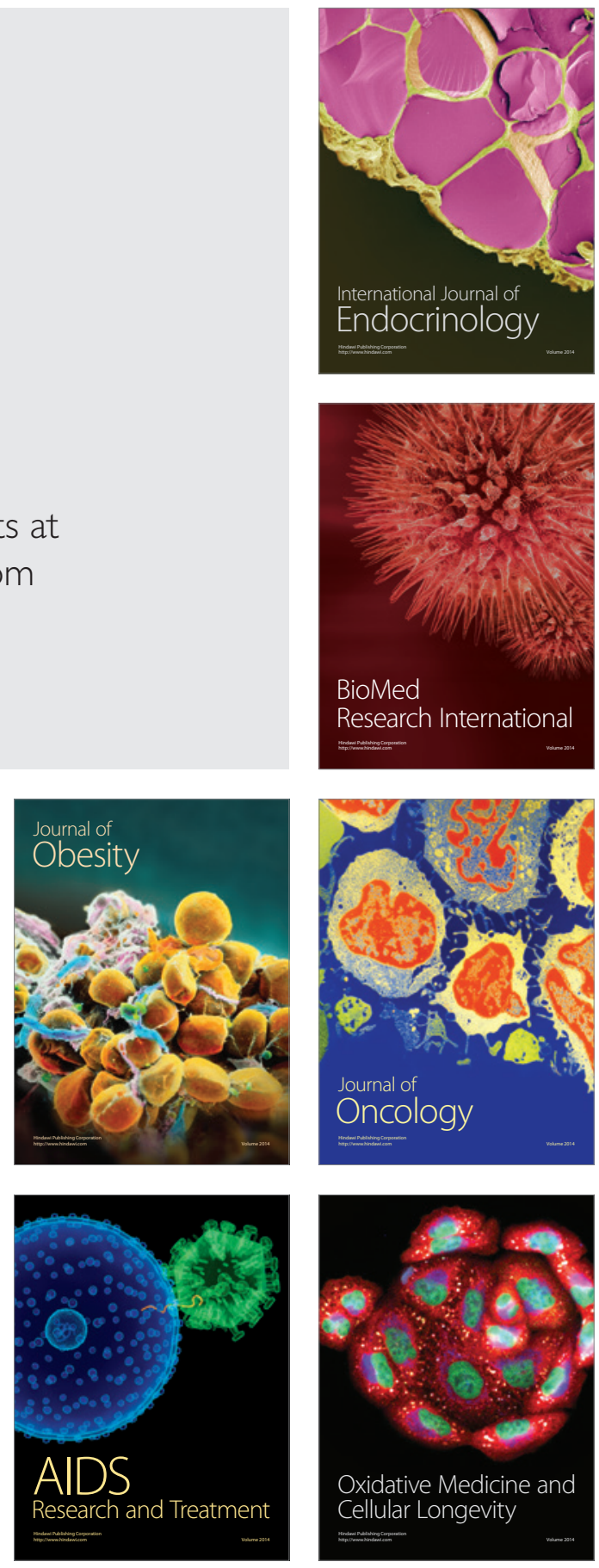